\title{
Organelle Genome Inheritance in Deparia Ferns (Athyriaceae, Aspleniineae, Polypodiales)
}

\author{
Li-Yaung Kuo ${ }^{\text {*t }}$, Te-Yen Tang ${ }^{2}$, Fay-Wei Li ${ }^{3,4}$, Huei-Jiun Su ${ }^{5}$, Wen-Liang Chiou ${ }^{2,6}$, \\ Yao-Moan Huang ${ }^{2 *}$ and Chun-Neng Wang ${ }^{1,7 *}$
}

${ }^{1}$ Institute of Ecology and Evolutionary Biology, National Taiwan University, Taipei, Taiwan, ${ }^{2}$ Taiwan Forestry Research Institute, Taipei, Taiwan, ${ }^{3}$ Boyce Thompson Institute, Ithaca, NY, United States, ${ }^{4}$ Plant Biology Section, Cornell University, Ithaca, NY, United States, ${ }^{5}$ Department of Earth and Life Sciences, University of Taipei, Taipei, Taiwan, ${ }^{6}$ Dr. Cecilia Koo Botanic Conservation Center, Pingtung, Taiwan, ${ }^{7}$ Department of Life Science, National Taiwan University, Taipei, Taiwan

\section{OPEN ACCESS}

Edited by:

Renchao Zhou,

Sun Yat-sen University, China

Reviewed by:

Yingjuan Su,

Sun Yat-sen University, China

Wei Lun Ng.

Sun Yat-sen University, China Yuchen Yang,

University of North Carolina at

Chapel Hill, United States

${ }^{*}$ Correspondence:

Li-Yaung Kuo

salvinia@hotmail.com

Yao-Moan Huang

huangym@tfri.gov.tw

Chun-Neng Wang

leafy@ntu.edu.tw

${ }^{\dagger}$ Present address:

Li-Yaung Kuo,

Boyce Thompson Institute, Ithaca, NY, United States

Specialty section:

This article was submitted to Evolutionary and Population Genetics, a section of the journal

Frontiers in Plant Science

Received: 24 January 2018

Accepted: 29 March 2018

Published: 13 April 2018

Citation:

Kuo L-Y, Tang T-Y, Li F-W, SU H-J,

Chiou $W-L$, Huang $Y-M$ and

Wang C-N (2018) Organelle Genome Inheritance in Deparia Ferns

(Athyriaceae, Aspleniineae,

Polypodiales). Front. Plant Sci. 9:486. doi: 10.3389/fpls.2018.00486
Organelle genomes of land plants are predominately inherited maternally but in some cases can also be transmitted paternally or biparentally. Compared to seed plants (> $83 \%$ genera of angiosperms and $>12 \%$ genera of gymnosperms), plastid genome (plastome) inheritance has only been investigated in fewer than $2 \%$ of fern genera, and mitochondrial genome (mitogenome) from only one fern genus. We developed a new and efficient method to examine plastome and mitogenome inheritance in a fern species-Deparia lancea (Athyriaceae, Aspleniineae, Polypodiales), and found that plastid and mitochondrial DNAs were transmitted from only the maternal parentage to a next generation. To further examine whether both organelle genomes have the same manner of inheritance in other Deparia ferns, we sequenced both plastid and mitochondrial DNA regions of inter-species hybrids, and performed phylogenetic analyses to identify the origins of organellar DNA. Evidence from our experiments and phylogenetic analyses support that both organelle genomes in Deparia are uniparentally and maternally inherited. Most importantly, our study provides the first report of mitogenome inheritance in eupolypod ferns, and the second one among all ferns.

Keywords: Deparia, eupolypod, fern, maternal inheritance, mitogenome, plastome, tissue-direct PCR

\section{INTRODUCTION}

Unlike the strict maternal inheritance of mitochondrial genomes in animals, organelle inheritance in land plants are complex and variable. Plant plastid and mitochondrial genomes (plastome and mitogenome, respectively) can be inherited maternally but also paternally or biparentally. In angiosperms, maternal inheritance is believed to be predominant in both organelle genomes; however, in around $20 \%$ of genera, plastomes were found to be putatively biparentally inherited (Mogensen, 1996; Zhang and Sodmergen, 2010; Jansen and Ruhlman, 2012; Choubey and Rajam, 2015). In a few angiosperm taxa, cases of biparental inheritance of the mitogenome and paternal inheritance of the plastome were confirmed (Zhang and Sodmergen, 2010; Li et al., 2013; Mccauley, 2013; Choubey and Rajam, 2015). In gymnosperms, both organelles are maternally inherited in non-conifer lineages, including cycads, Ginkgo, and gnetophytes (Mogensen, 1996; Jansen and Ruhlman, 2012). In contrast, for some conifers, paternal and biparental inheritance of the plastome, and paternal inheritance of the mitogenome was identified (Mogensen, 1996; Jansen and Ruhlman, 2012; Worth et al., 2014). 
In seed-free land plants, such as ferns and bryophytes, organelle genomes have been found to be only maternally inherited (reviewed in Zhang and Sodmergen, 2010). However, the variability of organelle genome inheritance for these plants is likely to be underestimated due to a poor and disproportional sampling. In ferns, while maternal inheritance is generally assumed, genetic evidence is weak-only four cases were studied for fern plastome inheritance, and only one was for that of mitogenome (Gastony and Yatskievych, 1992; Vogel et al., 1997; Guillon and Raquin, 2000; Adjie et al., 2007). The proportion of examined fern genera is less than 2\% (sensu PPG, 2016), and is far smaller than that in either angiosperms or gymnosperms (respectively estimated to be $>83 \%$ and $>12 \%$ of genera; Reboud and Zeyl, 1994; Mogensen, 1996; Zhang et al., 2003; Worth et al., 2014).

Cytologically, maternal inheritance of the organelle genomes in some ferns is implicated by the anatomical ontology during fertilization. Both plastids and mitochondria exist in fern egg cells and functional sperms (Duckett, 1973; Raghavan, 1989 and references listed therein; Kotenko, 1990; Gori et al., 1997; Muccifora et al., 2000; Renzaglia et al., 2001; Lopez-Smith and Renzaglia, 2002, 2008; Sakaushi et al., 2003; Cao et al., 2009, 2010; Wolniak et al., 2011; Cao, 2014), but plastids from sperm are known to be excluded before immersion into an egg (Bell and Duckett, 1976; Lopez-Smith and Renzaglia, 2002, 2008; Cao et al., 2010, 2016). In Osmunda, Pteridium, and Ceratopteris, mitochondria from sperms are digested soon after fertilization (Bell and Duckett, 1976; Lopez-Smith and Renzaglia, 2008; Cao et al., 2010, 2016). However, in Lygodium, paternal mitochondria are still retained after fertilization, and it is unclear whether these mitochondria persist in subsequent developmental stages (Lopez-Smith and Renzaglia, 2002).

This study aims to broaden the understanding of organelle genome inheritance in ferns by exploring an additional and uninvestigated lineage-Deparia (Athyriaceae, Aspleniineae, Polypodiales). Most importantly, we provide the second confirmed case of maternal inheritance of mitogenome in ferns, and the first one for that in the eupolypods, the most species-rich lineage in ferns (PPG, 2016). In addition, we developed a new genetic experiment (Figures 1, 2) that can greatly facilitate the investigations on organelle inheritance in seed-free plants like ferns, which mostly produce small and hermaphroditic gametophytes, and therefore, are difficult to be manipulated in outcrossing experiments to trace organelle genome transmissions. Given that ferns belong to the extant lineage most closely related to seed plants, an improved understanding of ferns should provide valuable insights into the evolution of variable organelle genome inheritance in land plants.

\section{MATERIALS AND METHODS}

\section{Overview of Experimental Design}

We developed a new genetic-based method to trace organelle genome transmission in ferns (Figures 1, 2). First, we selected two conspecific and sexual individuals (given names of " $A$ " and " $B$ " in Figure 2) from different populations as our parental sources. Next, we mixed their spores, and cultivated their gametophytes together until F1 sporophyte offspring were generated. At the same time, we applied a genome skimming approach (Figure $\mathbf{1}$ blue part) to seek single-nucleotide polymorphisms (SNPs) among their plastomes and mitogenomes, and found a plastid (pt)DNA marker and a mitochondrial (mt)DNA marker that can distinguish the two parental individuals. We also screened some candidate nuclear ( $n$ )DNA loci to find a diagnostic region as a biparentally inherited nDNA marker. For every individual of F1 sporophyte progenies, we then used these genetic markers to determine (i) its maternity by barcoding its attached gametophyte (i.e., the donor of egg) and (ii) its paternity by the nDNA marker genotyping (Figures 1, 2). Since we confirmed both parentages for these individuals, we could identify the outcrossed ones (i.e., crossing between gametophytes from the different parental individuals; Figure 2), and select them for further ptDNA and mtDNA genotyping. Compared with that of the two parental individuals, these genotyping results could further infer the parentage(s) that transmitted these organelle genomes from a gametophyte generation to a next sporophyte generation.

\section{Sample Preparation}

Two diploid individuals of Deparia lancea from two localities in Taiwan were selected as parental sources: Kuo4046 from Taichung City and Kuo4294 from Taoyuan City, which are referred to as parents $\mathrm{A}$ and $\mathrm{B}$, respectively. Their ploidies were confirmed by flow cytometry following Kuo (2015). Spores of the two parents were collected from living materials cultivated in a greenhouse of the Taiwan Forestry Research Institute. Their fertile leaves were wrapped in weighing paper, and dried at room temperature for 2-3 days to allow spore release. The collected spores were subsequently stored in $0.5 \mathrm{~mL}$ microcentrifuge tubes under $4^{\circ} \mathrm{C}$ and used within 2 months.

\section{Organelle Genome Assembly}

DNA of both parents were extracted from their leaves by a modified CTAB procedure (Kuo, 2015), and then fragmented into an averages size of 500 bp using Covaris S2 (Covaris, Woburn, MA, United States). We constructed the Illumina libraries using NEBNext DNA Library Prep Master Mix Set (New England Biolabs, Ipswich, MA, United States), which were sequenced on Illumina MiSeq $(2 \times 300$ bp paired-end) producing 0.4$0.6 \mathrm{~Gb}$ per sample. We removed the adapter sequences using Scythe (Buffalo, 2014) and trimmed reads to remove low quality bases by Sickle (Joshi and Fass, 2011). To assemble the organelle genomes, we input the reads into NOVOPlasty (Dierckxsens et al., 2017). For the plastome, we used the Woodwardia assembly (GenBank accession: NC_028543) as the seed. For the mitogenome, we used the coding exon sequences from Salvinia mitogenome ( $\mathrm{Li}$ et al., unpublished data) as the seeds, and used the Deparia plastome assemblies to exclude plastome reads (by 


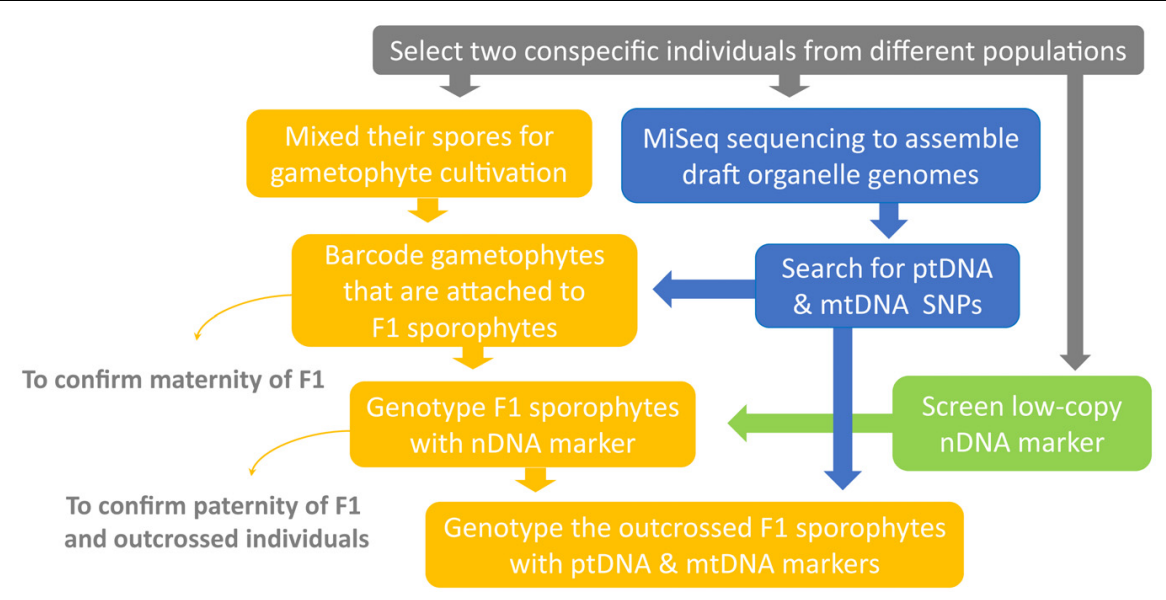

FIGURE 1 | Experimental workflow of the current study.

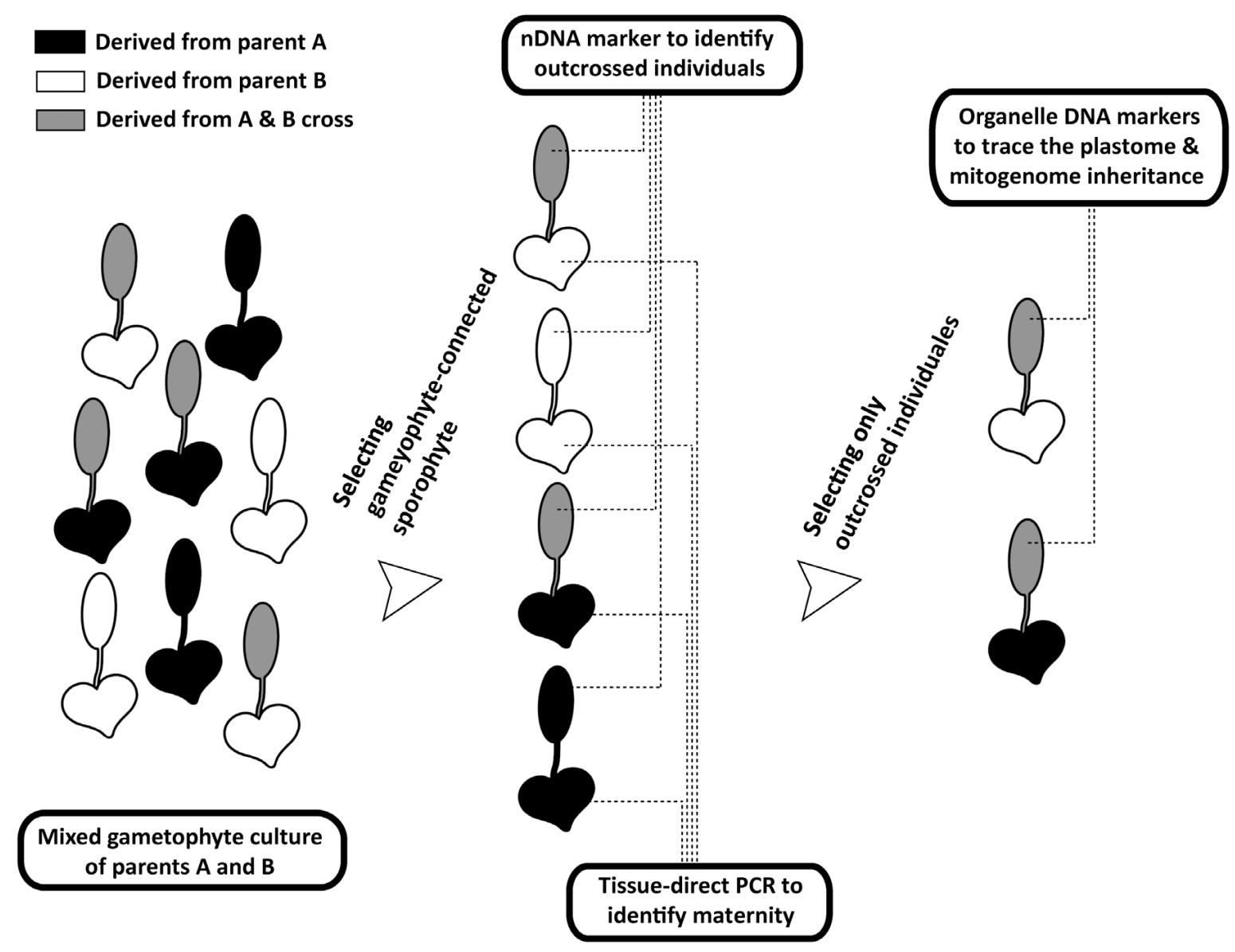

FIGURE 2 | Procedures of tracing organelle genome transmission in the current study. The heart- and spoon-shaped icons respectively indicate gametophyte and sporophyte juveniles of Deparia lancea. The white and black ones respectively indicate descendants from parent A or B, while the gray ones indicate outcrossed sporophytes between parents A and B.

"Chloroplast sequence $=$ " setting). The assembled organelle contigs were validated by read-mapping using bwa ( $\mathrm{Li}$ and Durbin, 2009), and annotated in Geneious (Kearse et al., 2012).
These raw reads were deposited in NCBI Short Read Archive (SRP136489) and the NCBI accessions for organelle contigs are: MH124207-35. 


\section{SNP Identification}

To identify SNPs in organelle genomes between the two parental individuals, we re-mapped their Illumina reads to our plastome and mitogenome assemblies using bwa (Li and Durbin, 2009), and then inspected the mapping results in Geneious. We found SNPs in the plastid $n d h \mathrm{~F}$ (NADH-plastoquinone oxidoreductase subunit five) and mitochondrial nad9 (NADH dehydrogenase subunit nine) genes (Supplementary Figures S1, S2), which were then respectively selected as the ptDNA marker and the mtDNA marker. Primers were designed to target these two regions (Table 1).To find a nDNA marker, we screened the low-copy loci from Rothfels et al. (2013), and found a diagnostic SNP at the 13th intron of IBR3 (IBA-response 3) (Supplementary Figure S3). To verify the sequences of the two IBR3 alleles in each parental individual, we used HiFi DNA polymerase (Kapa Biosystems, Wilmington, DE, United States) for PCR amplification, and cloned the PCR products into the pJET1.2/blunt cloning vector (Thermo Fisher Scientific, Waltham, MA, United States). Ligation, transformation, plating, and selection of clones followed the manufacturer's protocol. All primer information is summarized in Table 1. All the generated sequences were deposited in GenBank (Accession Nos.: MG972633-40).

\section{Culture for Gametophyte and Sporophyte Progeny}

Spores from both parental individuals were mixed and sowed on the top of soil medium in a $7.5 \times 9-\mathrm{cm}$ plastic box (PHYTATRAY II ${ }^{\mathrm{TM}}$ no. P5929; Sigma, St. Louis, MO, United States) with a density of roughly $320 \sim 350$ spores $/ \mathrm{cm}^{2}$. The soil medium contained a mixture of vermiculite: peat: perlite in a 2:2:1 volume ratio. After 3 months when the number of sporophyte offspring seemed saturated and no newly generated sporophyte individual was found, we transferred the gametophyte-connected sporophytes into individual plots. Both gametophytes and sporophytes were cultured under LED white fluorescent illumination of $6.3 \pm 0.3 \mu$ mole $\mathrm{m}^{-2} \mathrm{~s}^{-1}$ for $10 \mathrm{~h} \mathrm{~d}^{-1}$, and the daily temperature ranged $20 \sim 28^{\circ} \mathrm{C}$. The humidity was monitored to avoid desiccation of the cultures.

\section{Confirming Sexual Reproduction}

We first checked the spore number per sporangium (S/S) of both parental individuals to infer their reproductive modes. In Deparia, $64 \mathrm{~S} / \mathrm{S}$ and $32 \mathrm{~S} / \mathrm{S}$ are respectively indicative of sexual and apomictic individuals (Kato et al., 1992). In addition, we conducted flow cytometric analyses of both the gametophyte and sporophyte offspring to confirm their relative nuclear genome size, as well as their reproductive mode (i.e., with sexual reproduction, gametophytes should have a genome size half that of sporophyte progeny). Twenty gametophyte individuals (each around $0.5 \mathrm{~cm}^{2}$ in size) without a juvenile sporophyte were used for the flow cytometric analysis to confirm the gametophyte genome size; while leaf tissues of sporophyte juveniles were used to confirm the sporophyte genome size. The flow cytometric method followed Kuo (2015).

\section{Determining Maternity of F1 Sporophyte Progeny}

To determine whether the gametophyte-attached sporophyte progeny was derived from parent $\mathrm{A}$ or $\mathrm{B}$, we used the partial $n d h \mathrm{~F}$ as the DNA marker and a PCR-RFLP (restriction fragment length polymorphism) approach for identification. The $n d h \mathrm{~F}$ products of these gametophytes were first amplified using tissuedirect PCR following $\mathrm{Li}$ et al. (2010). Then, 1 or $2 \mu \mathrm{L}$ from each of these PCR products was treated with $5 \mathrm{U}$ of the restriction enzyme of AciI (New England Biolabs, Ipswich, MA, United States) at $37^{\circ} \mathrm{C}$ for $60 \mathrm{~min}$ and then $65^{\circ} \mathrm{C}$ for $20 \mathrm{~min}$. The AciI-treated $n d h \mathrm{~F}$ products were subsequently examined by electrophoresis using $1 \times \mathrm{TBE}$ and a $1.5 \%$ agarose gel at $110 \mathrm{~V}$ for $45 \mathrm{~min}$. After electrophoresis, the gel was then stained in an ethidium bromide solution for $10 \mathrm{~min}$. The gametophytes from parent A would have two DNA fragments on an electrophoresis gel (at 130 and $156 \mathrm{bp}$; Supplementary Figure S1), while those from parent B would have a single undigested 286 bp band.

\section{Identification of Sporophyte Progeny Resulting From Outcrossing}

After the sporophyte offspring became mature and they were large enough to produce spores, their genomic DNAs were extracted, using a modified $\mathrm{CTAB}$ procedure following Kuo (2015). We amplified the IBR3 sequences from these extracted DNAs. We performed a PCR in $15-\mu \mathrm{L}$ volume reactions, including $20 \mathrm{ng}$ of genomic DNA, $1 \times \mathrm{PCR}$ buffer $200 \mu \mathrm{M}$ dNTP, 15 pmol of each primer, and $1 \mathrm{U}$ polymerase (ExPrime Taq DNA Polymerase; GENETBIO, Daejeon, Korea). Because there is one SNP in the 13th intron that can distinguish between parents $\mathrm{A}$ and $\mathrm{B}$ (at position 248 in Figure S3), we directly sequenced these IBR3 amplicons, and used the presence of a double-peak signal to identify sporophyte individuals resulting from intergametophytic outcrossing.

\section{Tracing Organelle Genome Inheritance Using ptDNA and mtDNA Markers}

Genotyping of the ptDNA and mtDNA markers was conducted only on those outcrossed sporophyte offspring, and the results were compared to those of the parents to confirm their organelle genome inheritance. The partial $n d h \mathrm{~F}$ and the partial nad9 (respectively as the ptDNA and mtDNA markers) were amplified from genomic DNAs of these sporophyte offspring. The PCRs were conducted as described in the previous section. A single band from $n d h \mathrm{~F}$ PCR-RFLP indicates that ptDNA was derived from parent $B$ (see the detailed method in "Determining Maternity of F1 Sporophyte Progeny"). On the other hand, when DNA fragments with the sizes of 130 and $156 \mathrm{bp}$ were found, we additionally sequenced these $n d h \mathrm{~F}$ products to discern that if they contained sequences from only parent A or from both parents. The nad 9 genotypes of outcrossed sporophyte offspring were determined by sequencing. 
TABLE 1 | Primer information.

\begin{tabular}{|c|c|c|c|c|}
\hline Primer & Target taxon & Genetic Region $^{a}$ & $5^{\prime}-3^{\prime}$ sequence & Reference \\
\hline DI IBR3 fCSI & Deparia lanceab & IBR3 & CAACAAACATITCCTGCTCAATCAG & This study \\
\hline DI IBR3 rPGR & Deparia lanceab & IBR3 & CAATGGTGGAGTCTTCCTGG & This study \\
\hline AT IBR fPDV & Athyriaceae $^{\mathrm{c}}$ & IBR3 & GCAATGACTGAACCAGATGTG & This study \\
\hline AT IBR rAER & Athyriaceae $^{c}$ & IBR3 & ATSTCTATCCCACGCTCAGC & This study \\
\hline De ndhF fCGK & Deparia lanceab & $n d h F$ & GGGGACTTAATTGTGGAAAGG & This study \\
\hline Del ndhF rPSL & Depariac & $n d h F$ & CCATAAGGGATAAACTAAGCGAAG & This study \\
\hline Del nad9 fFAl & Deparia lanceab & nad9 & ATGACTTGCAGTCCACTTGAATAATTTGCTATTG & This study \\
\hline Del ndh9 rPWR & Deparia lanceab & nad9 & GGACGGCATTAGTCGCCAAGG & This study \\
\hline FernLr1 & Depariac & $\operatorname{trnL-L-F}$ & GGCAGCCCCCAGATTCAGGGGAACC & Li et al., 2011 \\
\hline f & Plants ${ }^{\mathrm{C}}$ & $\operatorname{trnL-L-F}$ & ATTGAACTGGTGACACGAG & Taberlet et al., 1991 \\
\hline FERpl2 fTFF & Ferns $^{\mathrm{C}}$ & rp/2 intron & CACCTIITCCGATGTCAC & This study \\
\hline De rpl2 rGGD & Deparia $^{c}$ & rp/2 intron & GGCGTAGTCTCCTCCAG & This study \\
\hline
\end{tabular}

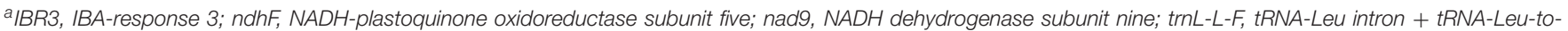
tRNA-Phe intergenic spacer; rpl2, ribosomal protein L2. ' ${ }^{b}$ Specific primer only for Deparia lancea. ${ }^{c}$ Universal primer.

\section{Organelle Genome Inheritance in Other Deparia Ferns}

To reveal possible manners of organelle genome inheritance in other Deparia ferns, we reconstructed both ptDNA and mtDNA phylogenies for some hybrids between the AT and DE clades (i.e., D. $\times$ tomitaroana, D. $\times$ nakaikeana, and D. $\times$ lobatocreneta; Kuo et al., 2018). By identifying the phylogenetic origins of their ptDNA and mtDNA, we can confirm whether these DNAs came from one or both parentages during hybridizations. trnL-L-F (including $t r n \mathrm{~L}$ gene and $t r n \mathrm{~L}-\mathrm{F}$ intergenic spacer) and $r p l 2$ intron were selected to reconstruct their ptDNA and mtDNA phylogenies, respectively. In total, ten $D . \times$ tomitaroana, one D. $\times$ nakaikeana, one D. $\times$ lobatocreneta individual, and 11 additional Deparia species (three from DE clade, four from AT clade, and one representative from each of the other clades; sensu Kuo et al., 2018) were sampled. Woodwardia radicans and Oceaniopteris gibba from Blechnaceae were selected as outgroups. We followed Li et al. (2011) for amplification and sequencing of $t r n \mathrm{~L}-\mathrm{L}-\mathrm{F}$, and for $r p l 2$ intron new primers were designed (Table 1). The voucher information and GenBank accessions for these samples are in Supplementary Table S1. The maximum likelihood phylogenies of these genes were reconstructed using IQ-TREE web server (Trifinopoulos et al., 2016) with 1000 ultrafast bootstrap replicates.

\section{RESULTS}

\section{Confirmation of Sexual Reproduction and Random Mating}

In both parental individuals of Deparia lancea, we found that only 64-spored sporangia were developed. Like other Deparia ferns (Kato et al., 1992), 64-spored sporangia indicate sexual reproduction, and produce spores that are half the ploidy level. Flow cytometric results further confirmed that these gametophytes had half the genome size compared to their sporophyte offspring (Figure 3). No indication of apomixis was found in the sampled individuals.

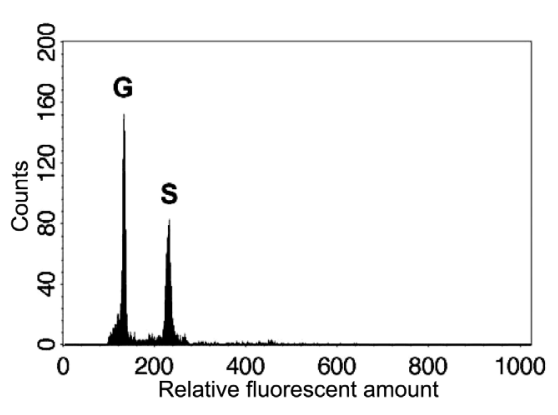

FIGURE 3 | Relative genome sizes of the gametophyte (G) and sporophyte (S) of the diploid Deparia lancea examined by flow cytometry.

In total, we genotyped 65 pairs of gametophyte and F1 sporophyte offspring. Overall, the resulting sporophyte offspring displayed a pattern of random mating, in which the observed numbers of outcrossed and selfed individuals (Table 2) do not significantly deviate from the expected numbers (Chi-squared $p>0.90$ ). Among the outcrossed individuals, 13 had $\mathrm{A}$ as the maternal parent and 11 had B (Table 2); and this result revealed no significant bias in maternal parentage (Chi-squared $p>0.60$ ), which further suggests that neither asymmetric mating nor cytonuclear incompatibility likely occurred among infraspecific and homoploidy crossings in Deparia lancea.

TABLE 2 | Genotyping results of sporophyte F1 offspring.

\begin{tabular}{|c|c|c|c|c|}
\hline F1 offspring ${ }^{a}$ & $\begin{array}{l}\text { Maternal } \\
\text { parent }^{\mathbf{b}}\end{array}$ & $\begin{array}{c}\text { Offspring } \\
\text { ptDNA }\end{array}$ & $\begin{array}{c}\text { Offspring } \\
\text { mtDNA }\end{array}$ & $\begin{array}{l}\text { Number of } \\
\text { individuals }\end{array}$ \\
\hline Outcrossed & A & Parent $A^{b}$ & Parent $A^{C}$ & 13 \\
\hline Outcrossed & $B$ & Parent B $\mathrm{B}^{\mathrm{b}, \mathrm{c}}$ & Parent Bc & 11 \\
\hline A selfed & A & - & - & 3 \\
\hline B selfed & B & - & - & 38 \\
\hline
\end{tabular}

aConfirmed by IBR3 sequences. ${ }^{b}$ Confirmed by ndhF PCR-RFLP. ${ }^{c}$ Confirmed by sequencing. 


\section{Identification of Outcrossed Progeny and ptDNA and mtDNA Genotype}

The numbers of outcrossed and inbred progenies are summarized in Table 2. In total, $37 \%$ of the sporophyte offspring were identified as outcrossing between parents A and B. Among the outcrossed offspring, 54 and $46 \%$ individuals had a maternal parent of $A$ and of $B$, respectively. All their ptDNA and mtDNA genotypes are same as those of their maternal parents.

\section{ptDNA and mtDNA Phylogenies}

The alignment of plastid trnL-L-F contained 889 characters with $25 \%$ of variable sites, and that of mitochondrial rpl2 intron contained 1384 characters with $1.4 \%$ of variable sites. The phylogenies inferred from these two regions are shown in Figure 4. In both phylogenies, all D. $\times$ tomitaroana individuals and $D . \times$ nakaikeana are nested in the DE clade. For D. $\times$ lobatocreneta, it is nested in the AT clade. In every of these hybrid individuals, we found ptDNA and mtDNA were inherited uniparentally from a same clade. In other words, ptDNA and mtDNA have the same manner of inheritance in these Deparia ferns.

\section{DISCUSSION}

\section{Advantages and Limitations of the Current Approach}

To directly infer organelle genome inheritance in land plants, artificial crosses and subsequent verification of the genomic constitution in the progenies have been commonly used. However, carrying out directional crosses with specific maternity and paternity is very difficult in ferns. Unlike seed plants, ferns are mostly homosporous (Haufler et al., 2016) and we cannot easily manipulate the gametophyte sex to ensure that sperms are coming from one gametophyte to the other. Moreover, it is almost impossible to infer organelle genome transmission in ferns by examining the organelle identity of germ line cells (e.g., eggs or sperms). Generally, mitochondria and plastids are present in both their sperms (or spermatocytes) and eggs (Duckett, 1973; Raghavan, 1989 and references therein; Kotenko, 1990; Gori et al., 1997; Muccifora et al., 2000; Renzaglia et al., 2001; Lopez-Smith and Renzaglia, 2002, 2008; Sakaushi et al., 2003; Cao et al., 2009, 2010; Wolniak et al., 2011; Cao, 2014 and references therein), thus any manner of organelle inheritance-maternal, paternal, and even biparentalis possible.

To overcome the difficulty of ascertaining maternity and paternity involved in a cross in fern species, two approaches were previously adopted. One common approach is to manipulate female and male gametophyte pairings for outcrossing (i.e., a hybrid between gametophyte individuals from different parents). In practice, such experiments need to first confirm the sex of the gametophyte individuals based on a gametangium observation, then separately select female and male individuals from sexmixed gametophyte cultures of identified sources, and pair them for outcrossing (e.g., Lovis, 1968; Guillon and Raquin, 2000; Yatabe et al., 2001). The genetic compositions of these resulting hybrids are further confirmed by biparentally inherited genetic markers (i.e., nuclear DNA markers) to exclude inbred progeny from outcrossed ones, because cultured female or male gametophytes might subsequently become bisexual. Such a method was used to trace plastome inheritance in several previous studies (Vogel et al., 1997; Guillon and Raquin, 2000; Adjie et al., 2007; Table 3). In the other approach (Gastony and Yatskievych, 1992), the paternal parentage of a fern hybrid species was identified as the sperm donor because this parental species was unable to produce functional eggs due to apomixis (reviewed in Gastony and Windham, 1989). The inheritance of organelle genomes in these hybrids were then revealed by ptDNA and mtDNA markers. However, these approaches mentioned above are labor-intensive for manipulating pairings of gametophyte individuals, or, for the latter case, have additional limitations requiring a cross between apomictic and sexual taxa.

Here, we develop a new approach which can efficiently confirm the maternity and paternity of an artificial cross/hybrid in ferns while avoiding manual pairing of gametophytes for outcrossing, and can be applied to most ferns and other seed-free plants as well. Because a sporophyte is initiated from a zygote inside an archegonium that is attached to its maternal gametophyte, we can genotype a gametophyte and the attached sporophyte in order to trace the maternal and paternal parentages (Figure 2). In this study, we determined the maternity of each sporophyte by barcoding its connected gametophytes using a genetic marker (i.e., ndhF PCR-RFLP). We then used nDNA marker (i.e., the SNP at 13th intron of IBR3) to identify the paternity. One key feature of our approach was the incorporation of tissue-direct PCR, which requires only a $>1 \mathrm{~mm}^{2}$ piece of tissue for a single PCR reaction (Li et al., 2010). This methodology enabled us to accomplish the DNA-based identification for gametophytes, thus verifying the maternal donor of sporophyte offspring. In addition, we adopted a genome skimming strategy to search SNPs throughout organelle genomes. Such strategy is more efficient, and better guarantees finding diagnostic regions for ptDNA and mtDNA markers. Especially for mtDNA, because of the limited genetic and genomic information for ferns (Guo et al., 2017) and slow substitution rate in plant (Gualberto and Newton, 2017), a strategy directly designing primers and seeking variable regions is usually not cost-effective.

Like other genetic-based approaches, this current approach requires the generation of outcrossed/hybridized F1 offspring from parental sources with preexisting genetic variation. Therefore, this approach is not applicable for plant species having no or very limited genetic variation at the population level. Another limitation of the current approach is that maternity identification of sporophyte offspring relies on DNA-barcoding the gametophyte which is attached on the sporophyte, and, as a result, female or bisexual gametophytes (i.e., egg donors) must generate with enough tissue amount for a barcoding experiment. Such requirement is very hard to be satisfied for the female gametophytes of heterosporous ferns and lycophytes 

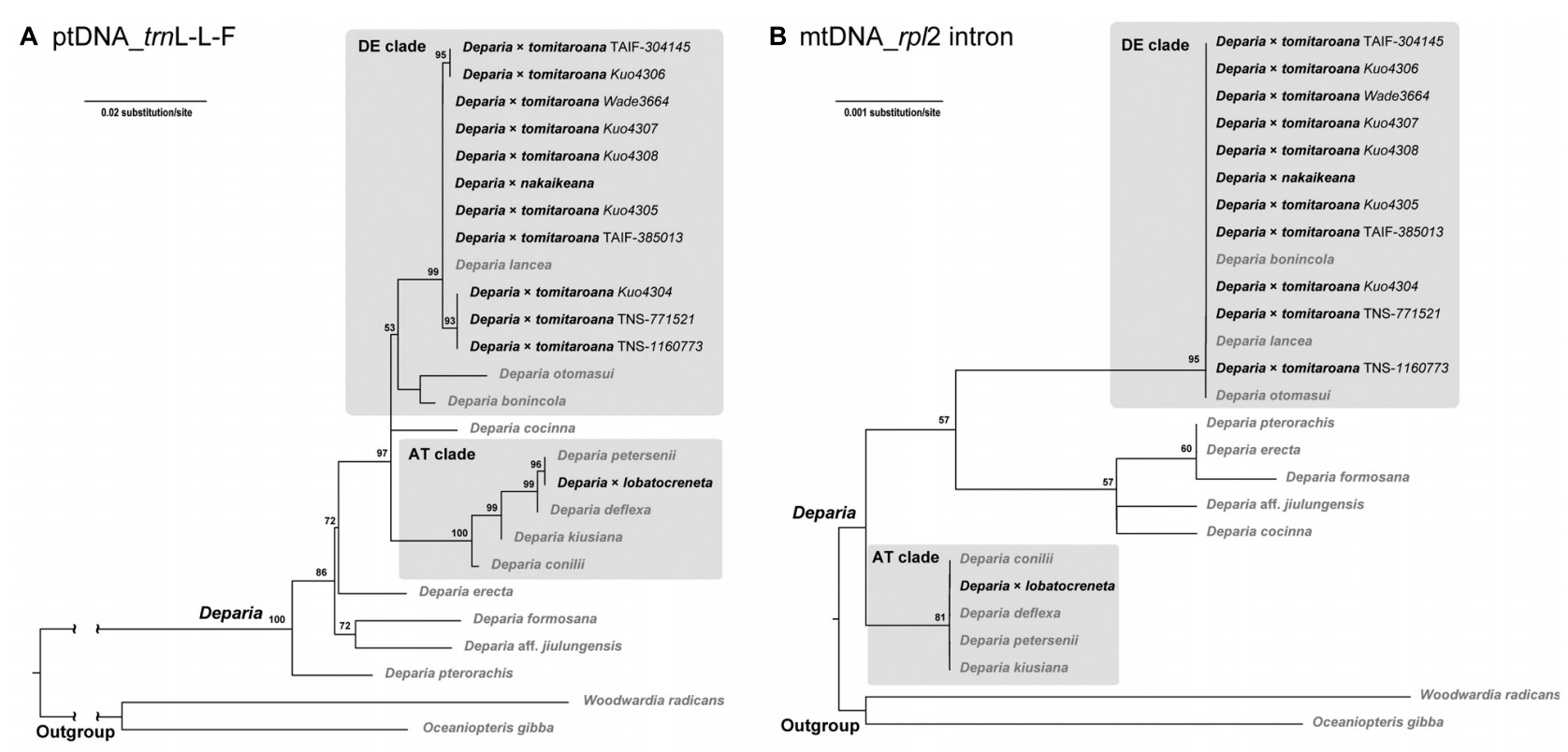

FIGURE 4 | (A) ptDNA (trnL-L-F) and (B) mtDNA (rp/2 intron) maximum likelihood phylogenies of the AT $\times$ DE inter-clade hybrids in Deparia. The values on branches are their bootstrap supports, and only the values larger than 50 are shown. The inter-clade hybrids are indicated with black names, and the other taxa are indicated with gray names.

TABLE 3 | Organelle genome inheritance in ferns.

\begin{tabular}{lllll}
\hline Genus (family) & Order/suborder & Organelle genome & Inheritance & Reference \\
\hline Equisetum (Equisetaceae) & Equisetales & Plastome & Maternal & Guillon and Raquin, 2000 \\
Ceratopteris (Pteridaceae) & Polypodiales/Pteridineae & Plastome & Maternal & Adjie et al., 2007 \\
Pellaea (Pteridaceae) & Polypodiales/Pteridineae & Plastome and mitogenome & Maternal & Gastony and Yatskievych, 1992 \\
Asplenium (Aspleniaceae) & Polypodiales/Aspleniineae & Plastome & Maternal & Vogel et al., 1997 \\
Deparia (Athyriaceae) & Polypodiales/Aspleniineae & Plastome and mitogenome & Maternal & This study \\
\hline
\end{tabular}

because these gametophytes are usually endosporous, minute, and composed of a limited number of cells (Raghavan, 1989). Fortunately, because these seed-free plants are heterosporous, their outcrossing can be easily manipulated by coculturing microspores (i.e., only developing into male gametophytes) and megaspores (i.e., only developing into female gametophytes) from two different individuals - a similar way as done for that of the seed plants.

\section{Organelle Genomes Inheritance in Deparia and Other Ferns}

In this study, we confirmed that plastome and mitogenome are inherited maternally in Deparia lancea (Table 2). After sequencing the ptDNA in nearly a half of the outcrossed individuals and mtDNA genotypes in all outcrossed individuals, we found no signal indicating paternal transmission of these genomes (Table 2). Based on our mtDNA and ptDNA phylogenies, we further confirmed in the inter-clade Deparia hybrids that their plastome and mitogenome inheritance are both uniparental (Figure 4), and are most likely maternal. The current study is the second to report mitogenome inheritance in ferns, and the first one in the most diversified fern lineage-the euploypods (Aspleniineae + Polypodiineae). For Aspleniineae plastome, our result is second to Vogel et al. (1997) to support maternal inheritance. In addition, our findings support one of the most important assumptions in fern phylogeny and genetics that ptDNA and mtDNA can only trace the maternal lineage.

Despite the scant case studies, our results show the consistency of maternal inheritance of the organelle genomes in ferns (Table 3). In addition, based on Chang et al. (2009), both plastome and mitogenome are likely maternally inherited in Dryopteris ferns (including Acrorumohra; Dryopteridaceae, Polypodiineae, Polypodiales). Given that apomictic fern gametophytes can only produce sperm cells (Gastony and Windham, 1989), Dryopteris diffracta, which produces 32-spored sporangia and is indicated as an apomictic species, should be the paternal parent of $D . \times$ subreflexipinna, while $D$. hasseltii should be the maternal parent. In Chang et al. (2009), they found all individuals of $D . \times$ subreflexipinna had identical ptDNA and mtDNA sequences to those of $D$. hasseltii. Taken together, so far, there is no known exception of maternal inheritance of the 
organelle genome in ferns as well as in seed-free land plants (Table 3; Zhang and Sodmergen, 2010). For land plant organelle genomes, biparental inheritance and RNA-editing are both suggested as important mechanisms to rescue deleterious DNA mutations or effects due to nucleocytoplasmic incompatibility (Zhang and Sodmergen, 2010; Castandet and Araya, 2012). It is worthwhile to further examine whether biparental inheritance possibly evolved as an alternative rescue mechanism in the seedfree plants known with no or relatively infrequent RNA-editing, such as Osmundales and Marattiales ferns (Knie et al., 2016). The results from these plants will better shed light on whether paternal transmission or biparental inheritance is restricted in seed plants.

\section{AUTHOR CONTRIBUTIONS}

L-YK designed and carried out the experiments, analyzed the data, and prepared the manuscript. T-YT assisted on the experiments. F-WL and H-JS assisted on genome assembling, and SNP identification. W-LC, Y-MH, and C-NW discussed the experimental design and results, and provided the facilities to accomplish this work. All authors commented and revised the manuscript.

\section{REFERENCES}

Adjie, B., Masuyama, S., Ishikawa, H., and Watano, Y. (2007). Independent origins of tetraploid cryptic species in the fern Ceratopteris thalictroides. J. Plant Res. 120, 129-138. doi: 10.1007/s10265-006-0032-5

Bell, P. R., and Duckett, J. G. (1976). Gametogenesis and fertilization in Pteridium. Bot. J. Linn. Soc. 73, 47-78. doi: 10.1111/j.1095-8339.1976.tb02012.x

Buffalo, V. S. (2014). A Bayesian Adapter Trimmer (Version 0.994 BETA). Available at https://github.com/vsbuffalo/scythe

Cao, J.-G. (2014). "The cytological studies of oogenesis and fertilization of ferns," in Reproductive Biology of Plants, eds K. G. Ramawat, J.-M. Mérillon, and K. R. Shivanna (Boca Raton, FL: CRC Press), 85-96. doi: 10.1201/b16535-5

Cao, J.-G., Dai, X.-F., Dai, X.-L., and Wang, Q.-X. (2009). An ultrastructural study of the mature spermatozoid of the fern Pteris fauriei. J. Chin. Electron. Microsc. Soc. 28, 420-425.

Cao, J.-G., Dai, X.-F., Dai, X.-L., and Wang, Q.-X. (2016). Observations on fertilization and a novel cytological mechanism for preventing polyspermy in the fern Osmunda japonica. Int. J. Plant Sci. 177, 287-293. doi: 10.1086/68 4523

Cao, J.-G., Wang, Q.-X., Yang, N.-Y., and Bao, W.-M. (2010). Cytological events during zygote formation of the fern Ceratopteris thalictroides. J. Integr. Plant Biol. 52, 254-264. doi: 10.1111/j.1744-7909.2010.00893.x

Castandet, B., and Araya, A. (2012). The nucleocytoplasmic conflict, a driving force for the emergence of plant organellar RNA editing. IUBMB Life 64, 120-125. doi: 10.1002/iub.581

Chang, H.-M., Chiou, W.-L., and Wang, J.-C. (2009). Molecular evidence for genetic heterogeneity and the hybrid origin of Acrorumohra subreflexipinna from Taiwan. Am. Fern J. 99, 61-77. doi: 10.1640/0002-8444-99.2.61

Choubey, A., and Rajam, M. V. (2015). "Organellar genomes of flowering plants," in Plant Biology and Biotechnology, eds B. Bahadur, M. Venkat Rajam, L. Sahijram, and K. Krishnamurthy (Berlin: Springer), 179-204. doi: 10.1007/978-81-3222283-5_8

Dierckxsens, N., Mardulyn, P., and Smits, G. (2017). NOVOPlasty: de novo assembly of organelle genomes from whole genome data. Nucleic Acids Res. 45:e18. doi: 10.1093/nar/gkw955

Duckett, J. G. (1973). An ultrastructural study of the differentiation of the spermatozoid of Equisetum. J. Cell Sci. 12, 95-129.

\section{FUNDING}

The main funding from Ministry of Science and Technology of Taiwan was granted to C-NW (MOST 106-2621-B-002 -002 -MY2) and Y-MH (MOST 106-2621- B-054- 002).

\section{ACKNOWLEDGMENTS}

We thank Chia-Wen Ko for maintaining the gametophyte and sporophyte cultures; Yu-Ling Hung, Hsin-Yi Huang, Bo-Hong Yeh, and Hong-Wen Ma for helping with DNA extraction; Riva Chu from Technology Common (College of Life Science, National Taiwan University, Taiwan) for the assistance with DNA library constructions for MiSeq; three reviewers for providing comments on the manuscript; and Daniel P. Chamberlin for helping with English editing.

\section{SUPPLEMENTARY MATERIAL}

The Supplementary Material for this article can be found online at: https://www.frontiersin.org/articles/10.3389/fpls.2018.00486/ full\#supplementary-material

Gastony, G., and Windham, M. (1989). Species concepts in pteridophytes: the treatment and definition of agamosporous species. Am. Fern J. 79, 65-77. doi: $10.2307 / 1547161$

Gastony, G., and Yatskievych, G. (1992). Maternal inheritance of the chloroplast and mitochondrial genomes in cheilanthoid ferns. Am. J. Bot. 79, 716-722. doi: 10.1002/j.1537-2197.1992.tb14613.x

Gori, P., Muccifora, S., Woo, S. L., and Bellani, L. M. (1997). An ultrastructural study of the mature spermatozoid of the fern Asplenium trichomanes L. subsp. trichomanes. Sex. Plant Reprod. 10, 142-148. doi: 10.1007/s004970050081

Gualberto, J. M., and Newton, K. J. (2017). Plant mitochondrial genomes: dynamics and mechanisms of mutation. Annu. Rev. Plant Biol. 68, 225-252. doi: 10.1146/ annurev-arplant-043015-112232

Guillon, J. M., and Raquin, C. (2000). Maternal inheritance of chloroplasts in the horsetail Equisetum variegatum (Schleich.). Curr. Genet. 37, 53-56. doi: 10.1007/s002940050008

Guo, W., Zhu, A., Fan, W., and Mower, J. P. (2017). Complete mitochondrial genomes from the ferns Ophioglossum californicum and Psilotum nudum are highly repetitive with the largest organellar introns. New Phytol. 213, 391-403. doi: $10.1111 /$ nph. 14135

Haufler, C. H., Pryer, K. M., Schuettpelz, E., Sessa, E. B., Farrar, D. R., Moran, R., et al. (2016). Sex and the single gametophyte: revising the homosporous vascular plant life cycle in light of contemporary research. Bioscience 66, 928-937. doi: 10.1093/biosci/biw108

Jansen, R. K., and Ruhlman, T. A. (2012). "Plastid genomes of seed plants," in Genomics of Chloroplasts and Mitochondria, eds R. Bock and V. Knoop (Netherlands: Springer), 103-126. doi: 10.1007/978-94-007-2920-9

Joshi, N. A., and Fass, J. N. (2011). Sickle: A Sliding-Window, Adaptive, QualityBased Trimming Tool for FastQ Files. Available at: github.com/najoshi/ sickle

Kato, M., Nakato, N., Cheng, X., and Iwatsuki, K. (1992). Cytotaxonomic study of ferns of Yunnan, southwestern China. Bot. Mag. 105, 105-124. doi: 10.1007/ BF02489407

Kearse, M., Moir, R., Wilson, A., Stones-Havas, S., Cheung, M., Sturrock, S., et al. (2012). Geneious basic: an integrated and extendable desktop software platform for the organization and analysis of sequence data. Bioinformatics 28 , 1647-1649. doi: 10.1093/bioinformatics/bts199

Knie, N., Grewe, F., Fischer, S., and Knoop, V. (2016). Reverse U-to-C editing exceeds C-to-U RNA editing in some ferns - a monilophyte-wide comparison 
of chloroplast and mitochondrial RNA editing suggests independent evolution of the two processes in both organelles. BMC Evol. Biol. 16:134. doi: 10.1186/ s12862-016-0707-z

Kotenko, J. (1990). Spermatogenesis in a homosporous fern. Onoclea sensibilis. Am. J. Bot. 77, 809-825. doi: 10.1002/j.1537-2197.1990.tb14470.x

Kuo, L.-Y. (2015). Polyploidy and Biogeography in Genus Deparia and Phylogeography in Deparia lancea. Ph.D. dissertation, National Taiwan University, Taipei.

Kuo, L.-Y., Ebihara, A., Kato, M., Rouhan, G., Ranker, T. A., Wang, C.-N., et al. (2018). Morphological characterization of infra-generic lineages in Deparia (Athyriaceae: Polypodiales). Cladistics 34, 78-92. doi: 10.1111/cla.12192

Li, D., Qi, X., Li, X., Li, L., Zhong, C., and Huang, H. (2013). Maternal inheritance of mitochondrial genomes and complex inheritance of chloroplast genomes in Actinidia Lind.: evidences from interspecific crosses. Mol. Genet. Genomics 288, 101-110. doi: 10.1007/s00438-012-0732-6

Li, F.-W., Kuo, L.-Y., Huang, Y.-M., Chiou, W.-L., and Wang, C.-N. (2010). Tissuedirect PCR, a rapid and extraction-free method for barcoding of ferns. Mol. Ecol. Resour. 10, 92-95. doi: 10.1111/j.1755-0998.2009.02745.x

Li, F.-W., Kuo, L.-Y., Rothfels, C. J., Ebihara, A., Chiou, W.-L., Windham, M. D., et al. (2011). rbcL and matK earn two thumbs up as the core DNA barcode for ferns. PLoS One 6:e26597. doi: 10.1371/journal.pone.0026597

Li, H., and Durbin, R. (2009). Fast and accurate short read alignment with BurrowsWheeler transform. Bioinformatics 25, 1754-1760. doi: 10.1093/bioinformatics/ btp324

Lopez-Smith, R., and Renzaglia, K. (2008). Sperm cell architecture, insemination, and fertilization in the model fern, Ceratopteris richardii. Sex. Plant Reprod. 21, 153-167. doi: 10.1007/s00497-008-0068-x

Lopez-Smith, R., and Renzaglia, K. S. (2002). "Comparisons between pre-released and swimming sperm cells of the fern Lygodium japonicum," in Botany 2002 Botany in the Curriculum, 4-7 August Abstracts (Madison, WI: Botanical Society of America).

Lovis, J. D. (1968). Fern hybridists and fern hybridising II. Fern hybridising at the University of Leeds. Br. Fern Gaz. 10, 13-20.

Mccauley, D. E. (2013). Paternal leakage, heteroplasmy, and the evolution of plant mitochondrial genomes. New Phytol. 200, 966-977. doi: 10.1111/nph.xyb12431

Mogensen, H. (1996). The hows and whys of cytoplasmic inheritance in seed plants. Am. J. Bot. 3, 383-404. doi: 10.1002/j.1537-2197.1996.tb12718.x

Muccifora, S., Woo, S. L., and Gori, P. (2000). Ultrastructural features of spermatocytes and spermatozoids in the fern Phyllitis scolopendrium (L.) Newm. subsp. scolopendrium. Sex. Plant Reprod. 12, 323-331. doi: 10.1007/ s004970000024

PPG, I. (2016). A community-derived classification for extant lycophytes and ferns. J. Syst. Evol. 54, 563-603. doi: 10.1111/jse.12229

Raghavan, V. (1989). Developmental Biology of Fern Gametophyte, eds P. W. Barlow, D. Bray, P. B. Green, and J. M. W. Slack. New York, NY: Cambridge University Press. doi: 10.1017/CBO9780511529757

Reboud, X., and Zeyl, C. (1994). Organelle inheritance in plants. Heredity 72, 132-140. doi: 10.1038/hdy.1994.19

Renzaglia, K. S., Johnson, T. H., Gates, H. D., and Whittier, D. P. (2001). Architecture of the sperm cell of Psilotum. Am. J. Bot. 88, 1151-1163. doi: $10.2307 / 3558326$
Rothfels, C. J., Larsson, A., Li, F.-W., Sigel, E. M., Huiet, L., Burge, D. O., et al. (2013). Transcriptome-mining for single-copy nuclear markers in ferns. PLoS One 8:e76957. doi: 10.1371/journal.pone.0076957

Sakaushi, S., Okoshi, M., Miyamura, S., and Hori, T. (2003). Swimming behavior and ultrastructure of sperm of Lygodium japonicum (Pteridophyta). Sex. Plant Reprod. 16, 113-122. doi: 10.1007/s00497-0030184-6

Taberlet, P., Gielly, L., Pautou, G., and Bouvet, J. (1991). Universal primers for amplification of three non-coding regions of chloroplast DNA. Plant Mol. Biol. 17, 1105-1109. doi: 10.1007/BF00037152

Trifinopoulos, J., Nguyen, L. T., von Haeseler, A., and Minh, B. Q. (2016). W-IQ-TREE: a fast online phylogenetic tool for maximum likelihood analysis. Nucleic Acids Res. 44, W232-W235. doi: 10.1093/nar/ gkw256

Vogel, J. C., Russell, S. J., Rumsey, F. J., Barrett, J. A., and Gibby, M. (1997). ). Evidence for maternal transmission of chloroplast DNA in the genus Asplenium (Aspleniaceae, Pteridophyta). Bot. Acta 111, 247-249. doi: 10.1111/j.1438-8677. 1998.tb00704.x

Wolniak, S. M., van der Weele, C. M., Deeb, F., Boothby, T., and Klink, V. P. (2011). Extremes in rapid cellular morphogenesis: post-transcriptional regulation of spermatogenesis in Marsilea vestita. Protoplasma 248, 457-473. doi: 10.1007/ s00709-011-0276-3

Worth, J. R. P., Yokogawa, M., and Isagi, Y. (2014). Outcrossing rates and organelle inheritance estimated from two natural populations of the Japanese endemic conifer Sciadopitys verticillata. J. Plant Res. 127, 617-626. doi: 10.1007/s10265014-0646-y

Yatabe, Y., Masuyama, S., Darnaedi, D., and Murakami, N. (2001). Molecular systematics of the Asplenium nidus complex from Mt. Halimun National Park, Indonesia: evidence for reproductive isolation among three sympatric rbcL. Am. J. Bot. 88, 1517-1522. doi: 10.2307/3558459

Zhang, Q., Liu, Y., and Sodmergen (2003). Examination of the cytoplasmic DNA in male reproductive cells to determine the potential for cytoplasmic inheritance in 295 angiosperm species. Plant Cell Physiol. 44, 941-951. doi: 10.1093/pcp/ pcg121

Zhang, Q., and Sodmergen (2010). Why does biparental plastid inheritance revive in angiosperms? J. Plant Res. 123, 201-206. doi: 10.1007/s10265-0090291-z

Conflict of Interest Statement: The authors declare that the research was conducted in the absence of any commercial or financial relationships that could be construed as a potential conflict of interest.

The reviewers WN and YS and handling Editor declared their shared affiliation.

Copyright (C) 2018 Kuo, Tang, Li, Su, Chiou, Huang and Wang. This is an open-access article distributed under the terms of the Creative Commons Attribution License (CC BY). The use, distribution or reproduction in other forums is permitted, provided the original author(s) and the copyright owner are credited and that the original publication in this journal is cited, in accordance with accepted academic practice. No use, distribution or reproduction is permitted which does not comply with these terms. 